Research Paper

\title{
The Effect of Marital Status on Nasopharyngeal Carcinoma Survival: A Surveillance, Epidemiology and End Results Study
}

\author{
San-Gang Wu ${ }^{1^{*}}$, Qing-Hong Zhang ${ }^{2^{*}}$, Wen-Wen Zhang ${ }^{3^{*}}$, Jia-Yuan Sun ${ }^{3}$, Qin Lin ${ }^{\circledR}{ }^{\bowtie}$, Zhen-Yu He ${ }^{{ }^{凶}}$ \\ 1. Department of Radiation Oncology, Xiamen Cancer Hospital, The First Affiliated Hospital of Xiamen University, Xiamen 361003, People's Republic of \\ China; \\ 2. Department of Anesthesiology, The First Affiliated Hospital of Xiamen University, Xiamen 361003, People's Republic of China; \\ 3. Department of Radiation Oncology, Sun Yat-sen University Cancer Center, State Key Laboratory of Oncology in South China, Collaborative Innovation \\ Center of Cancer Medicine, Guangzhou 510060, People's Republic of China. \\ *San-Gang Wu, Qing-Hong Zhang, and Wen-Wen Zhang contributed equally to this work.
}

$\square$ Corresponding authors: Qin Lin, Department of Radiation Oncology, Xiamen Cancer Hospital, The First Affiliated Hospital of Xiamen University, Xiamen 361003, People's Republic of China; Tel: +86 592 2139531; Fax: +86 592 2137301; E-mail. linqin9531@126.com and Zhen-Yu He, Department of Radiation Oncology, Sun Yat-sen University Cancer Center, State Key Laboratory of Oncology in South China, Collaborative Innovation Center of Cancer Medicine, 651 Dongfeng Road East, Guangzhou 510060, People’s Republic of China; Tel: +86 20 87343543; Fax: +86 2087343392 E-mail. hezhy@sysucc.org.cn

(C) Ivyspring International Publisher. This is an open access article distributed under the terms of the Creative Commons Attribution (CC BY-NC) license (https://creativecommons.org/licenses/by-nc/4.0/). See http://ivyspring.com/terms for full terms and conditions.

Received: 2017.11.21; Accepted: 2018.01.29; Published: 2018.04.27

\begin{abstract}
Purpose: This study examined the role of marital status on survival outcome of nasopharyngeal carcinoma (NPC) patients using a population-based cancer registry.

Methods: Patients with primary NPC diagnosed between 2004 and 2013 were included using the Surveillance, Epidemiology and End Results program. Patient demographic, clinicopathologic features, management, and survival outcomes were compared according to marital status. Cause-specific survival (CSS, NPC-related death) for marital status was analyzed.

Results: The data of 3018 patients were included, with $61.4 \%, 11.1 \%, 21.8$, and $5.6 \%$ of patients married, divorced (or separated), single, and widowed, respectively. Widowed patients had the highest proportion of elderly age $(p<0.001)$, were more likely to be female $(p<0.001)$, and had more well-to-moderately differentiated $(p<0.001)$ and node-negative disease $(p=0.038)$. Widowed patients were also less likely to have received radiotherapy and chemotherapy compared with patients of other marital status $(p<$ 0.001 ). The 5 -year CSS was $76.1 \%, 70.8 \%, 73.4 \%$, and $59.8 \%$ in the married, divorced, single, and widowed groups, respectively $(p=0.001)$. Marital status was the independent prognostic factor for CSS. Widowed patients had a significantly increased risk of NPC-related death compared with married (hazard ratio $[\mathrm{HR}]$ 2.014, 95\% confidence interval [Cl] 1.477-2.747, $p<0.001)$, divorced ( $\mathrm{HR} 1.580,95 \% \mathrm{Cl}$ 1.087-2.295, $p=0.017)$, and single (HR 2.000, 95\% Cl 1.402-2.854, $p<0.001)$ patients. The divorced ( $p$ $=0.067)$ and single $(p=0.949)$ groups had similar CSS to the married group.

Conclusions: Being widowed was associated with an increased the risk of cancer mortality in NPC compared with being married, divorced, or single.
\end{abstract}

Key words: Nasopharyngeal carcinoma, Marital status, SEER, Survival, Prognosis

\section{Introduction}

Nasopharyngeal carcinoma (NPC) is a rare malignancy in most countries around the world, including the United States (US), with an incidence of $<1$ case per 100000 person-years (1). However, NPC is epidemic in Asian countries, especially in southern
China, with an incidence of nearly 20-50/100 000 person-years $(1,2)$. The Chinese population has become one of the fastest-growing immigrant populations in the US in recent decades, and Chinese people continue to be the population most affected by NPC, 
accounting for $42.7 \%$ of NPC patients in the US (3).

Radiotherapy and chemotherapy are the main treatments for NPC. However, pretreatment nutritional status, serious side effects including weight loss and acute oral mucosa during radiotherapy and chemotherapy, and radiation-related sequelae, which may negatively affect patient survival and quality of life (4-7). Social support is an important component of patient screening, treatment, and follow-up care. It has been suggested that having a spouse encourages early screening and compliance with treatment, improving outcome. Therefore, social situation is important to patients with and survivors of NPC. The available evidence suggests that married patients with cancer tend to be diagnosed at an earlier stage and are more likely to receive recommended or aggressive therapy $(8,9)$. If social support is the primary beneficial effect of marriage, the protective effects of marriage may be particularly important for survival in NPC.

Marital status has been found to be related to survival outcome in various cancers (8-15). A recent population-based study identified the 10 leading causes of cancer death, including head and neck cancer, and the results also indicated that unmarried patients (i.e. those separated, divorced, widowed, or never married) tended to be diagnosed with advanced disease and to be undertreated, and had a higher risk of early cancer-related death than their married counterparts (9). One Surveillance, Epidemiology and End Results (SEER) study of head and neck cancer found that marriage had a protective effect on survival of oral and laryngeal cancer but not oropharyngeal, hypopharyngeal, or nasopharyngeal cancers. However, the study only classified patients as married or unmarried (16). A SEER-Medicare study found that marriage was associated with better outcomes in oral cavity and pharyngeal cancers. However, in this study only $12.4 \%$ of the patients had NPC, and they were not further classified by type of unmarried status (such as never married, separated, divorced, or widowed) (15). Previous research, including several studies investigating thyroid, ovarian, uterine, and gastric cancers $(12,13,17,18)$, has found that widowed patients are at greater risk of cancer mortality. However, to date, limited studies have specifically focused on the association between marital status and NPC outcomes (19). In this study, we investigated the role of marital status in NPC survival using a SEER-based population.

\section{Materials and methods}

\section{Patients}

We identified NPC patients from the SEER program, which is maintained by the National Cancer Institute and represents approximately $28 \%$ of the US population (20). SEER registries provide accurate, timely, and continuous data on cancer incidence, patient demographics, and survival. Patients diagnosed with primary NPC between 2004 and 2013 were included. Patients with insufficient or unknown demographics, clinicopathologic, and treatment parameters were excluded.

\section{Covariates and outcome variables}

The following variables were identified: age, gender, race/ethnicity, grade, tumor stage (T-stage), nodal stage (N-stage), metastatic stage (M-stage), radiotherapy, and chemotherapy. Marital status information was gathered in four categories: married, single (never married), divorced (divorced or separated), and widowed. We included age group ( $\leq 50$ or $>50$ years), gender (male or female), race/ethnicity (White, Black, American Indian/ Alaskan Native, or Asian/Pacific Islander), grade (well differentiated, moderately differentiated, or poorly/undifferentiated), T-stage (T1, T2, T3, or T4), $\mathrm{N}$-stage (N0, N1, N2, or N3), M-stage (M0 or M1), radiotherapy (no or yes), and chemotherapy (no/unknown or yes) as covariates in the adjusted models. The primary survival outcome of this study was cause-specific survival (CSS), defined as time from initial diagnosis to the date of death due to NPC.

\section{Statistical analyses}

Patient demographics, clinicopathologic, and treatment characteristics were compared according to marital status. Frequencies were compared using the Chi-square exact test. Survival rate was expressed by Kaplan-Meier curve, and the survival differences were compared using the log-rank test. We calculated hazard ratios (HRs) and their 95\% confidence intervals (CIs) using the univariate and multivariate Cox proportional hazards regression models to assess survival differences between marital status categories. The variables with significant differences in the univariate analysis were entered in to multivariate regression models. All analyses were conducted using SPSS version 21 statistical software (IBM Corporation, Armonk, USA). A $p$-value $<0.05$ was considered statistically significant.

\section{Results}

We included 3018 patients with a median follow-up time of 31 months (range 0-119 months), and $80.4 \%$ of patients were still alive at the end of the study. Of the patients, $61.4 \%(\mathrm{n}=1854)$ were married, $11.1 \%(\mathrm{n}=338)$ were divorced, $21.8 \%(\mathrm{n}=657)$ were single, and $5.6 \%(n=169)$ were widowed. The 
baseline characteristics of patients are summarized in Table 1.

The widowed patients had the highest proportion of elderly age (>50 years) $(97.0 \%$ vs. $38.7-69.8 \%, p<0.001)$ and females $(65.1 \%$ vs. $25.3-31.7 \%, p<0.001$ ), and single patients tended to be younger than those of other marital status $(61.3 \%$ vs. $3.0-33.0 \%, p<0.001)$. Asian and Pacific Islander NPC patients were more likely to be married $(44.4 \%$ vs. 20.7-28.2\%, $p<0.001$ ), and more Black patients were single $(24.7 \%$ vs. $6.4-16.0 \%, p<0.001)$, while more divorced and widowed patients were found among White patients $(61.5-65.1 \%$ vs. $45.5-47.6 \%, p<0.001)$. Poorly/undifferentiated disease was highest in married and single patients, compared with divorced and widowed patients $(84.0-84.9 \%$ vs. $71.0-76.9 \%, p<$ 0.001). Early T-stage was highest among married patients $(36.7 \%$ vs. $26.0-33.1 \%, p<0.001)$, while widowed patients had the highest proportion of N0 stage $(36.7 \%$ vs. $25.6-29.1 \%, p=0.038)$. A significantly higher proportion of married patients received radiotherapy $(89.9 \%$ vs. $82.2-86.4 \%, p<0.001)$, while the proportion of widowed patients who received chemotherapy was significantly lower than the proportions of other marital status $(68.6 \%$ vs. $79.0-81.7 \%, p$ $<0.001)$. However, there was no significant difference in M-stage between the four marital status groups.

Married, divorced, and single patients with NPC had a superior CSS compared with their widowed counterparts $(p<0.001)$. Five-year CSS was $76.1 \%$, $70.8 \%, 73.4 \%$, and $59.8 \%$ in the married, divorced, single, and widowed groups, respectively (log-rank test, $p<0.001$ ) (Figure 1). Survival among married, divorced, and single groups was not significantly different (log-rank test, $p=0.133$ ). We reanalyzed married, divorced, and single patients versus the widowed group, and found no significant difference in T-stage $(p=0.986), \mathrm{N}$-stage $(p=0.105)$, or M-stage ( $p$ $=0.972$ ) in the non-widowed patients compared with the widowed group, while patients with widowed group had a lower incidence of node-positive disease $(p=0.015)$ compared with non-widowed patients.

Table 1. The baseline demographics, clinicopathologic, and treatment characteristics of 3018 nasopharyngeal carcinoma patients according to marital status.

\begin{tabular}{|c|c|c|c|c|c|c|}
\hline Variables & $\mathbf{n}$ & $\begin{array}{l}\text { Married } \\
(\%)\end{array}$ & Divorced (\%) & $\begin{array}{l}\text { Single } \\
(\%)\end{array}$ & Widowed (\%) & $p$ \\
\hline \multicolumn{7}{|l|}{ Age (years) } \\
\hline$\leq 50$ & 1122 & $612(33.0)$ & $102(30.2)$ & $403(61.3)$ & $5(3.0)$ & $<0.001$ \\
\hline$>50$ & 1896 & $1242(67.0)$ & $236(69.8)$ & $254(38.7)$ & $164(97.0)$ & \\
\hline \multicolumn{7}{|l|}{ Gender } \\
\hline Male & 2132 & $1385(74.7)$ & $231(68.3)$ & $457(69.6)$ & $59(34.9)$ & $<0.001$ \\
\hline Female & 886 & $469(25.3)$ & $107(31.7)$ & $200(30.4)$ & $110(65.1)$ & \\
\hline \multicolumn{7}{|l|}{ Race/ethnicity } \\
\hline White & 1506 & $883(47.6)$ & $220(65.1)$ & $299(45.5)$ & $104(61.5)$ & $<0.001$ \\
\hline Black & 348 & $119(6.4)$ & $40(11.8)$ & $162(24.7)$ & $27(16.0)$ & \\
\hline American Indian/Alaska Native & 43 & $28(1.5)$ & $1(0.3)$ & $11(1.7)$ & $3(1.8)$ & \\
\hline Asian or Pacific Islander & 1121 & $824(44.4)$ & $77(22.8)$ & $185(28.2)$ & $35(20.7)$ & \\
\hline \multicolumn{7}{|l|}{ Grade } \\
\hline Well differentiated & 92 & $55(3.0)$ & $10(3.0)$ & $19(2.9)$ & $8(4.7)$ & $<0.001$ \\
\hline Moderately differentiated & 430 & $241(13.0)$ & $68(20.1)$ & $80(12.2)$ & $41(24.3)$ & \\
\hline Poorly/undifferentiated & 2496 & $1558(84.0)$ & $260(76.9)$ & $558(84.9)$ & $120(71.0)$ & \\
\hline \multicolumn{7}{|l|}{ T-stage } \\
\hline $\mathrm{T} 1$ & 1015 & $680(36.7)$ & $108(32.0)$ & $171(26.0)$ & $56(33.1)$ & $<0.001$ \\
\hline $\mathrm{T} 2$ & 717 & $467(25.2)$ & $73(21.6)$ & $138(21.0)$ & $39(23.1)$ & \\
\hline $\mathrm{T} 3$ & 640 & $370(20.0)$ & $80(23.7)$ & $154(23.4)$ & $36(21.3)$ & \\
\hline $\mathrm{T} 4$ & 646 & $337(18.2)$ & $77(22.8)$ & $194(29.5)$ & $38(22.5)$ & \\
\hline \multicolumn{7}{|l|}{ N-stage } \\
\hline N0 & 860 & $540(29.1)$ & $90(26.6)$ & $168(25.6)$ & $62(36.7)$ & 0.038 \\
\hline N1 & 1001 & $634(34.2)$ & $116(34.3)$ & $202(30.7)$ & $49(29.0)$ & \\
\hline N2 & 793 & $474(25.6)$ & $86(25.4)$ & 195 (29.7) & $38(22.5)$ & \\
\hline N3 & 364 & $206(11.1)$ & 46 (13.6) & $92(14.0)$ & $20(11.8)$ & \\
\hline \multicolumn{7}{|l|}{ M-stage } \\
\hline M0 & 2712 & 1674 (90.3) & $311(92.0)$ & 575 (87.5) & $152(89.9)$ & 0.109 \\
\hline M1 & 306 & $180(9.7)$ & $27(8.0)$ & $82(12.5)$ & 17 (10.1) & \\
\hline \multicolumn{7}{|l|}{ Radiotherapy } \\
\hline No & 371 & $188(10.1)$ & $46(13.6)$ & $107(16.3)$ & $30(17.8)$ & $<0.001$ \\
\hline Yes & 2647 & 1666 (89.9) & $292(86.4)$ & $550(83.7)$ & 139 (82.2) & \\
\hline \multicolumn{7}{|l|}{ Chemotherapy } \\
\hline No/unknown & 584 & 339 (18.3) & $71(21.0)$ & $121(18.4)$ & $53(31.4)$ & $<0.001$ \\
\hline Yes & 2434 & 1515 (81.7) & $267(79.0)$ & $536(81.6)$ & $116(68.6)$ & \\
\hline
\end{tabular}

$\mathrm{N}$, nodal; $\mathrm{M}$, metastasis; $\mathrm{T}$, tumor. 


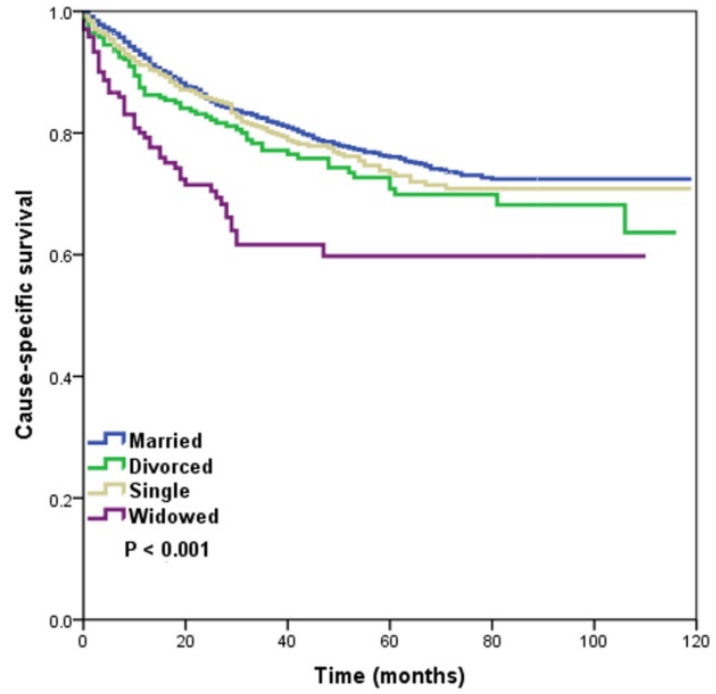

Figure 1. Kaplan-Meier survival curves in patients with nasopharyngeal carcinoma according to marital status
In the univariate analysis, age, gender, race/ethnicity, grade, $\mathrm{T}$-stage, $\mathrm{N}$-stage, $\mathrm{M}$-stage, radiotherapy, chemotherapy, and marital status were the prognostic factors of CSS (Table 2). In multivariate analysis, when controlling for the above variables, marital status was still the independent predictor for CSS. Widowed patients had a significantly increased risk of NPC-related death compared with married (HR 2.014, 95\% CI 1.477-2.747, $p<0.001$ ), divorced (HR 1.580, 95\% CI 1.087-2.295, $p=0.017$ ), and single (HR 2.000, 95\% CI 1.402-2.854, $p<0.001$ ) patients. The divorced group (HR 1.275, 95\% CI 0.983-1.654, $p=$ 0.067 ) and the single group (HR 1.007, 95\% CI $0.811-1.250, p=0.949$ ) had similar CSS compared to the married group. The other predictors independently associated with higher risk of death from NPC were increased age, male gender, well-differentiated disease, advanced T-stage and N-stage, and no receipt of radiotherapy and chemotherapy (Table 2).

Table 2. Univariate and multivariate analysis for evaluating the effect of marital status on nasopharyngeal carcinoma-specific survival.

\begin{tabular}{|c|c|c|c|c|c|c|}
\hline \multirow[t]{2}{*}{ Variables } & \multicolumn{3}{|c|}{ Univariate } & \multicolumn{3}{|c|}{ Multivariate } \\
\hline & HR & $95 \% \mathrm{CI}$ & $p$ & HR & $95 \% \mathrm{CI}$ & $p$ \\
\hline \multicolumn{7}{|l|}{ Age (years) } \\
\hline$\leq 50$ & 1 (ref) & & & 1 (ref) & & \\
\hline$>50$ & 1.576 & $1.324-1.875$ & $<0.001$ & 1.542 & $1.275-1.865$ & $<0.001$ \\
\hline \multicolumn{7}{|l|}{ Gender } \\
\hline Male & 1 (ref) & & & 1 (ref) & & \\
\hline Female & 0.769 & $0.638-0.928$ & 0.006 & 0.783 & $0.645-0.951$ & 0.014 \\
\hline \multicolumn{7}{|l|}{ Race/ethnicity } \\
\hline White & 1 (ref) & & & 1 (ref) & & \\
\hline Black & 0.981 & $0.756-1.272$ & 0.884 & 0.892 & $0.680-1.169$ & 0.407 \\
\hline American Indian/Alaska Native & 1.296 & $0.710-2.365$ & 0.399 & 1.093 & $0.595-2.008$ & 0.774 \\
\hline Asian or Pacific Islander & 0.818 & $0.685-0.976$ & 0.025 & 0.885 & $0.734-1.067$ & 0.200 \\
\hline \multicolumn{7}{|l|}{ Grade } \\
\hline Well differentiated & 1 (ref) & & & 1 (ref) & & \\
\hline Moderately differentiated & 0.804 & $0.520-1.242$ & 0.326 & 0.829 & $0.534-1.287$ & 0.404 \\
\hline Poorly/undifferentiated & 0.556 & $0.374-0.825$ & 0.004 & 0.638 & $0.426-0.955$ & 0.029 \\
\hline \multicolumn{7}{|l|}{ T-stage } \\
\hline $\mathrm{T} 1$ & 1 (ref) & & & 1 (ref) & & \\
\hline $\mathrm{T} 2$ & 1.382 & $1.079-1.770$ & 0.010 & 1.415 & $1.100-1.819$ & 0.007 \\
\hline $\mathrm{T} 3$ & 2.218 & $1.756-2.801$ & $<0.001$ & 2.195 & $1.726-2.791$ & $<0.001$ \\
\hline $\mathrm{T} 4$ & 2.998 & $2.395-3.754$ & $<0.001$ & 2.847 & 2.252-3.599 & $<0.001$ \\
\hline \multicolumn{7}{|l|}{ N-stage } \\
\hline No & 1 (ref) & & & 1 (ref) & & \\
\hline N1 & 0.955 & $0.769-1.186$ & 0.678 & 1.107 & $0.881-1.392$ & 0.383 \\
\hline N2 & 1.091 & $0.873-1.364$ & 0.442 & 1.238 & $0.975-1.572$ & 0.079 \\
\hline N3 & 1.905 & $1.486-2.441$ & $<0.001$ & 1.824 & $1.396-2.382$ & $<0.001$ \\
\hline \multicolumn{7}{|l|}{ M-stage } \\
\hline M0 & 1 (ref) & & & 1 (ref) & & \\
\hline M1 & 4.253 & $3.505-5.161$ & $<0.001$ & 2.922 & $2.366-3.610$ & $<0.001$ \\
\hline \multicolumn{7}{|l|}{ Radiotherapy } \\
\hline No & 1 (ref) & & & 1 (ref) & & \\
\hline Yes & 0.315 & $0.258-0.385$ & $<0.001$ & 0.437 & $0.348-0.548$ & $<0.001$ \\
\hline \multicolumn{7}{|l|}{ Chemotherapy } \\
\hline No/unknown & 1 (ref) & & & 1 (ref) & & \\
\hline Yes & 0.786 & $0.643-0.961$ & 0.019 & 0.756 & $0.598-0.957$ & $<0.001$ \\
\hline \multicolumn{7}{|l|}{ Marital status } \\
\hline Married & 1 (ref) & & & 1 (ref) & & \\
\hline Divorced & 1.286 & $0.993-1.665$ & 0.057 & 1.275 & $0.983-1.654$ & 0.067 \\
\hline Single & 1.099 & $0.897-1.348$ & 0.362 & 1.007 & $0.811-1.250$ & 0.949 \\
\hline Widowed & 2.224 & $1.652-2.993$ & $<0.001$ & 2.014 & $1.477-2.747$ & $<0.001$ \\
\hline
\end{tabular}

$\mathrm{CI}$, confidence interval; HR, hazard ratio; N, nodal; $\mathrm{M}$, metastasis; $\mathrm{T}$, tumor 
Table 3. Multivariate models to estimate the individual interaction on nasopharyngeal carcinoma-specific survival between marital status and gender, tumor stage and nodal stage.

\begin{tabular}{|c|c|c|c|c|}
\hline Variables & Married & Divorced HR (95\%CI) & Single HR $(95 \% \mathrm{CI})$ & Widowed HR (95\%CI) \\
\hline \multicolumn{5}{|l|}{ Gender } \\
\hline \multirow[t]{2}{*}{ Male } & 1 (ref) & $1.257(0.929-1.701)$ & $0.886(0.686-1.144)$ & $1.816(1.156-2.853)$ \\
\hline & & $p=0.138$ & $p=0.353$ & $p=0.01$ \\
\hline \multirow[t]{2}{*}{ Female } & 1 (ref) & $1.398(0.829-2.358)$ & $1.439(0.942-2.198)$ & $2.294(1.434-3.669)$ \\
\hline & & $p=0.209$ & $p=0.093$ & $p=0.001$ \\
\hline \multicolumn{5}{|l|}{ T-stage } \\
\hline \multirow[t]{2}{*}{$\mathrm{T} 1-\mathrm{T} 2$} & 1 (ref) & $1.414(0.949-2.105)$ & $1.169(0.827-1.652)$ & $2.203(1.367-3.550)$ \\
\hline & & $p=0.088$ & $p=0.376$ & $p=0.001$ \\
\hline \multirow[t]{2}{*}{ T3-T4 } & 1 (ref) & $1.134(0.804-1.599)$ & $0.973(0.736-1.285)$ & 1.778 (1.194-2.647) \\
\hline & & $p=0.473$ & $p=0.846$ & $p=0.005$ \\
\hline \multicolumn{5}{|l|}{ N-stage } \\
\hline \multirow[t]{2}{*}{ No } & 1 (ref) & $1.267(0.764-2.101)$ & $0.904(0.558-1.466)$ & $2.034(1.163-3.558)$ \\
\hline & & $p=0.359$ & $p=0.683$ & $p=0.013$ \\
\hline \multirow[t]{2}{*}{ N1-3 } & 1 (ref) & $1.267(0.933-1.720)$ & $1.072(0.840-1.370)$ & 1.891 (1.294-2.763) \\
\hline & & $p=0.129$ & $p=0.575$ & $p=0.001$ \\
\hline
\end{tabular}

CI, confidence interval; $\mathrm{HR}$, hazard ratio; $\mathrm{N}$, nodal; $\mathrm{T}$, tumor

Three multivariate Cox regression models were used to assess the individual interaction effects of marital status with gender, T-stage, and N-stage. Each model was adjusted for gender, race/ethnicity, grade, M-stage, radiotherapy, chemotherapy, and two of the three variables. Widowed patients remained at a higher risk of poor CSS compared with the non-widowed groups, in the three multivariate models (Table 3).

\section{Discussion}

In this study, we investigated the impact of marital status on survival outcome in NPC patients diagnosed between 2004 and 2013 based on a population-based cohort. Our results showed that widowed patients had a poor CSS compared with those who were married, divorced, or single.

The survival of NPC was related to disease stage in our study, which is consistent with the results of other studies from the endemic area of NPC $(21,22)$. Several studies have found that marriage was related to diagnosis at early-stage disease, with a higher proportion of distant disease found in unmarried groups (8-15). In a SEER study of 51272 head and neck cancer patients $(6.1 \%$ of whom had NPC), being unmarried was also associated with advanced tumor stage (16). However, our study showed that early T-stage was highest among married patients, while widowed patients comprised the highest proportion with N0 stage. As there was no survival difference between the married, divorced, and single groups in our study, we further reanalyzed married, divorced, or single versus widowed status, and did not find a significant difference in T-stage, $\mathrm{N}$-stage, or M-stage in the non-widowed patients compared with the widowed group. Approximately 50\% of patients in our study were at local advanced stage, which may be due to the difficulty of early diagnosis in NPC.
Therefore, family support may not have been associated with regular screening visits in NPC. Although the US is not an epidemic area for NPC, we still believe that family support plays an important role in the early diagnosis, treatment, and post-treatment care of NPC.

The difference in the extent of care provided may potentially affect patient outcome after cancer diagnosis. Previous studies have indicated that married patients were more likely to receive curative therapy and high-quality care, compared with widowed patients $(23,24)$. A SEER study that included 1260898 patients and 10 cancer sites showed that married patients tended to receive definitive therapy more than their unmarried (including never married, divorced, separated, and widowed) counterparts (9). In the current study, although widowed patients had favorable characteristics including node-negative disease and females compared to non-widowed patients. However, the married, divorced, and single patients were more likely to receive radiotherapy and chemotherapy than their widowed counterparts. The potential reason to explain the unfavorable prognosis of widower individuals is undertreatment. Several studies have indicated that single patients were more likely to have delayed treatment or refuse treatment than patients who were married $(25,26)$. However, further subgroup analysis of single patients was not performed in these studies.

A previous SEER study by Xu et al. (19) was also aimed to investigate the effect of marital status in NPC between 1973 and 2012, and the results indicated that unmarried patients had significantly poorer CSS and OS compared to married patients. However, the effect of marital status including married, divorced, single, and widowed were not further compared in the multivariate analysis, it may therefore be exaggerated the impact of marriage and underestimated the effects of the widowed patients. 
In addition, the $\mathrm{T}$-stage and $\mathrm{N}$-stage were also not identified due to the interval of the study spanned nearly 40 years. Moreover, the chemotherapy usage was not recorded in the study. In the present study, we only included patients with complete demographics, clinicopathologic and treatment parameters to minimize the potential impact of various parameters on the results, and our results indicated that patients with widowed group had poor CSS compared to patients with married, divorced, single.

The results of our study are consistent with those from previous studies of various cancer sites showing that widowed patients were at increased risk of cancer mortality $(12,13,17,18)$. The influence of marital status on survival outcomes can hypothetically be explained by psychosocial factors that are independent of clinicopathologic features and extent of treatment. From a physiologic standpoint, the mechanisms for investigating the effect of psychosocial factors on survival in cancer have yielded many reasonable explanations. The association between marital status and cancer-related death is often described in terms of the positive effects of the marital relationship, which may provide socially supportive actions including encouraging spouses in healthy behavior and in pursuing curative treatment, and helping to reduce anxiety and stress during the course of and after treatment (27). In addition, several studies have proposed that increased psychological stress and decreased psychosocial support may impair the immune response and contribute to tumor progression by activating the hypothalamic-pituitary-adrenal axis. This regulates the release of glucocorticoids and catecholamines, which further directly influences the tumor microenvironment $(28,29)$ and has been implicated in cancer survival (29-32).

In our study, more widowed patients were female. It has been observed that the amount of natural killer cells is significantly reduced in women whose husbands have recently died, compared with controls (33). In addition, significantly higher rates of severe depression have been found in divorced and widowed patients (34). It is intuitive that decreased social support may seriously impaired cellular immune function, and a higher level of social support would enhance the cells responsible for recognizing and killing cancer cells by activating natural killer cell activity $(35,36)$. Although most of the previous studies were performed with non-NPC patients, the physiologic mechanisms of the effects of marital status on survival may be similar. These data support the suggestion of a link between social support, marriage, and immune responses such as natural killer cell activity. Our analyses consistently showed that widowed patients had the worst outcome in cancer-related death, and the survival benefit previously attributed to marriage is explained by the inferior survival of widows.

The limitations of our study should be considered. First, the SEER program only records marital status at the time of diagnosis; whether marital status changes after diagnosis is not reported. Such change may also affect survival and confound the difference in survival outcomes according to marital status. Second, there may be a considerable proportion of patients who are not legally married, but live in de facto same- or opposite-sex partnerships. This may contribute to an underestimate of the true impact of social support on survival outcomes. Third, detailed information about radiotherapy, chemotherapy, disease recurrence, and comorbidities was lacking in the SEER database. In addition, the SEER database had recorded a high specificity of data for radiotherapy and chemotherapy. However, overall sensitivity was $80 \%$ and $68 \%$ for radiotherapy and chemotherapy data, respectively (37). Finally, the results of our study can only be generalized to the US population and are not representative of the global population, especially of NPC in epidemic areas.

\section{Conclusion}

In conclusion, our results suggest that being widowed increases the risk of NPC mortality, when compared with NPC patients who are married, divorced, or single. Our study demonstrates that widowed patients are a newly identified high-risk subgroup with significantly poorer prognosis, who may potentially benefit from seeking social support and personalized care in the absence of a marital relationship. The targeted incorporation of social support into the integrated treatment and personalized care of NPC patients should be a national healthcare priority for providers and policymakers.

\section{Competing Interests}

The authors have declared that no competing interest exists.

\section{References}

1. Jemal A, Bray F, Center MM, et al. Global cancer statistics. CA Cancer J Clin. 2011;61(2):69-90.

2. Wei KR, Zheng RS, Zhang SW, et al. Nasopharyngeal carcinoma incidence and mortality in China in 2010. Chin J Cancer. 2014;33(8):381-387.

3. Ou SH, Zell JA, Ziogas A, Anton-Culver H. Epidemiology of nasopharyngeal carcinoma in the United States: improved survival of Chinese patients within the keratinizing squamous cell carcinoma histology. Ann Oncol. 2007;18(1):29-35.

4. Hong JS, Hua YJ, Su L, et al. Modified-Nutrition Index is a Significant Prognostic Factor for the Overall Survival of the Nasopharyngeal Carcinoma 
Patients who Undergo Intensity-modulated Radiotherapy. Nutr Cancer 2017;69(7):1011-1018.

5. Li K, Yang L, Hu QY, et al. Oral Mucosa Dose Parameters Predicting Grade $\geq 3$ Acute Toxicity in Locally Advanced Nasopharyngeal Carcinoma Patients Treated With Concurrent Intensity-Modulated Radiation Therapy and Chemotherapy: An Independent Validation Study Comparing Oral Cavity versus Mucosal Surface Contouring Techniques. Transl Oncol. 2017;10(5):752-759

6. Zhang W, Chen Y, Chen L, et al. Importance of maintaining body weight for prevention of distant metastasis of nasopharyngeal carcinoma: An alternative workflow for cancer-risk assessment. J Cancer. 2017;8(12):2269-2276.

7. Chan JW, Parvathaneni U, Yom SS. Reducing radiation-related morbidity in the treatment of nasopharyngeal carcinoma. Future Oncol. 2017;13(5):425-431.

8. Goodwin JS, Hunt WC, Key CR, et al. The effect of marital status on stage, treatment, and survival of cancer patients. JAMA. 1987;258(21):3125-3130.

9. Aizer AA, Chen $\mathrm{MH}, \mathrm{McCarthy} \mathrm{EP}$, et al. Marital status and survival in patients with cancer. J Clin Oncol. 2013;31(31):3869-3876.

10. Nayeri K, Pitaro G, Feldman JG. Marital status and stage at diagnosis in cancer. N Y State J Med.1992;92(1):8-11.

11. Gore JL, Kwan L, Saigal CS, et al. Marriage and mortality in bladder carcinoma. Cancer. 2005;104(6):1188-1194.

12. Mahdi H, Kumar S, Munkarah AR, et al. Prognostic impact of marital status on survival of women with epithelial ovarian cancer. Psychooncology. 2013;22(1):83-88.

13. Lowery WJ, Stany MP, Phippen NT, et al. Survival advantage of marriage in uterine cancer patients contrasts poor outcome for widows: a Surveillance, Epidemiology and End Results study. Gynecol Oncol. 2015;136(2):328-335.

14. El Ibrahimi S, Pinheiro PS. The effect of marriage on stage at diagnosis and survival in women with cervical cancer. Psychooncology. 2017;26(5):704-710.

15. Schaefer EW, Wilson MZ, Goldenberg D, et al. Effect of marriage on outcomes for elderly patients with head and neck cancer. Head Neck. 2015;37(5):735-742.

16. Inverso G, Mahal BA, Aizer AA, et al. Marital status and head and neck cancer outcomes. Cancer. 2015;121(8):1273-1278.

17. Shi RL, Qu N, Lu ZW, et al. The impact of marital status at diagnosis on cancer survival in patients with differentiated thyroid cancer. Cancer Med. 2016;5(8):2145-2154.

18. Zhou R, Yan S, Li J. Influence of marital status on the survival of patients with gastric cancer. J Gastroenterol Hepatol. 2016;31(4):768-775.

19. Xu C, Liu X, Chen YP, et al. Impact of marital status at diagnosis on survival and its change over time between 1973 and 2012 in patients with nasopharyngeal carcinoma: a propensity score-matched analysis. Cancer Med. 2017;6(12):3040-3051

20. [Internet] National Cancer Institute. Surveillance, Epidemiology, and End Results (SEER) Program SEER*Stat Database: Incidence - SEER 18 Regs Custom Data (with chemotherapy recode), Nov 2015 Sub (2000-2013) $<$ Katrina/Rita Population Adjustment $>$ - Linked To County Attributes - Total U.S., 1969-2014 Counties, National Cancer Institute, DCCPS, Surveillance Research Program, released July 2016, based on the November 2015 submission. www.seer.cancer.gov

21. OuYang PY, Xiao Y, You KY, et al. Validation and comparison of the 7th and 8th edition of AJCC staging systems for non-metastatic nasopharyngeal carcinoma, and proposed staging systems from Hong Kong, Guangzhou, and Guangxi. Oral Oncol. 2017;72:65-72.

22. Pan JJ, Ng WT, Zong JF, et al. Prognostic nomogram for refining the prognostication of the proposed 8th edition of the AJCC/UICC staging system for nasopharyngeal cancer in the era of intensity-modulated radiotherapy. Cancer. 2016;122(21):3307-3315.

23. Denberg TD, Beaty BL, Kim FJ, et al. Marriage and ethnicity predict treatment in localized prostate carcinoma. Cancer. 2005;103(9):1819-1825.

24. Iwashyna TJ, Christakis NA. Marriage, widowhood, and health-care use. Soc Sci Med. 2003;57(11):2137-2147.

25. Naghavi AO, Echevarria MI, Strom TJ, et al. Treatment delays, race, and outcomes in head and neck cancer. Cancer Epidemiol. 2016;45:18-25.

26. Massa ST, Osazuwa-Peters N, Franco J, et al. Survival after refusal of surgical treatment for locally advanced laryngeal cancer. Oral Oncol. 2017;71:34-40.

27. Pinquart M, Duberstein PR. Associations of social networks with cancer mortality: a meta-analysis. Crit Rev Oncol Hematol. 2010;75(2):122-137.

28. Sood AK, Bhatty R, Kamat AA, et al. Stress hormone-mediated invasion of ovarian cancer cells. Clin Cancer Res. 2006;12(2):369-375.

29. Antoni MH1, Lutgendorf SK, Cole SW, et al. The influence of bio-behavioural factors on tumour biology: pathways and mechanisms. Nat Rev Cancer. 2006;6(3):240-248.

30. Garssen B, Goodkin K. On the role of immunological factors as mediators between psychosocial factors and cancer progression. Psychiatry Res. 1999;85(1):51-61.

31. Sklar LS, Anisman H. Stress and coping factors influence tumor growth. Science. 1979;205(4405):513-515.

32. Reiche EM, Nunes SO, Morimoto HK. Stress, depression, the immune system, and cancer. Lancet Oncol. 2004:5(10):617-625.

33. Irwin M, Daniels $\mathrm{M}$, Risch SC, et al. Plasma cortisol and natural killer cell activity during bereavement. Biol Psychiatry. 1988;24(2):173-178.

34. Chou KL, Cheung KC. Major depressive disorder in vulnerable groups of older adults, their course and treatment, and psychiatric comorbidity. Depress Anxiety. 2013;30(6):528-537.
35. Levy SM, Herberman RB, Whiteside T, et al. Perceived social support and tumor estrogen/progesterone receptor status as predictors of natural killer cell activity in breast cancer patients. Psychosom Med. 1990;52(1):73-85.

36. Lutgendorf SK, Sood AK, Anderson B, et al. Social support, psychological distress, and natural killer cell activity in ovarian cancer. J Clin Oncol. 2005;23(28):7105-7113.

37. Noone AM, Lund JL, Mariotto A, et al. Comparison of SEER Treatment Data With Medicare Claims. Med Care. 2016;54(9):e55-64. 